\title{
BMJ Open Vasohibin-1 expression as a biomarker of aggressive nature in ductal adenocarcinoma of the prostate: a retrospective cohort study at two centres in Japan
}

\author{
Hiroaki Kobayashi, ${ }^{1,2}$ Takeo Kosaka (D) , ${ }^{1}$ Shuji Mikami, ${ }^{3}$ Tokuhiro Kimura, ${ }^{4}$ \\ Hiroshi Hongo, ${ }^{1}$ Michio Kosugi, ${ }^{2}$ Yasufumi Sato, ${ }^{5}$ Mototsugu Oya ${ }^{1}$
}

To cite: Kobayashi H, Kosaka T, Mikami S, et al. Vasohibin-1 expression as a biomarker of aggressive nature in ductal adenocarcinoma of the prostate: a retrospective cohort study at two centres in Japan. BMJ Open 2021;11:e056439. doi:10.1136/ bmjopen-2021-056439

- Prepublication history and additional supplemental material for this paper are available online. To view these files, please visit the journal online (http://dx.doi.org/10.1136/ bmjopen-2021-056439).

Received 16 August 2021 Accepted 01 November 2021

Check for updates

(C) Author(s) (or their employer(s)) 2021. Re-use permitted under CC BY-NC. No commercial re-use. See rights and permissions. Published by BMJ.

For numbered affiliations see end of article.

Correspondence to

Dr Takeo Kosaka;

takemduro@gmail.com

\section{ABSTRACT}

Objectives Vasohibin-1 (VASH1) is an endogenous angiogenesis regulator expressed in activated vascular endothelial cells. We previously reported that high VASH1 expression is a predictor of progression in acinar adenocarcinoma of the prostate. In this study, we evaluated the characteristics of ductal adenocarcinoma of the prostate by comparing the level of VASH1 expression between ductal and acinar adenocarcinoma specimens.

Design and setting A retrospective cohort study at two centres in Japan.

Participants Among the 1495 patients who underwent radical prostatectomy or transurethral resection for the past 15 years, a total of 14 patients diagnosed with ductal adenocarcinoma and 20 patients diagnosed with acinar adenocarcinoma with a Gleason score of $4+4$ were included.

Interventions We immunohistochemically examined the CD34 expression as the microvessel density (MVD) and activated endothelial cells as the VASH1 density (vessels per $\mathrm{mm}^{2}$ ).

Primary and secondary outcome measures The primary outcome was the association of MVD and VASH1 density between ductal and acinar adenocarcinoma, and the secondary outcome was their oncological outcomes. Results Nine patients (64.3\%) with ductal adenocarcinoma were diagnosed at an advanced clinical stage, and five patients $(35.7 \%)$ died from cancer during a median follow-up of 56.0 months. The VASH1 densities $($ mean $\pm S D)$ in ductal and acinar adenocarcinoma were $45.1 \pm 18.5$ vs $16.1 \pm 21.0(p<0.001)$, respectively, while the MVD (mean $\pm S D$ ) in ductal and acinar adenocarcinoma were $65.3 \pm 21.9$ vs $80.8 \pm 60.7$ ( $p=0.666)$, respectively. The 5-year cancer-specific survival rates for high and low VASH1 expression were $70.0 \%$ and $100.0 \%(p=0.006)$, respectively. High VASH1 expression and a diagnosis of ductal adenocarcinoma were significant predictors of cancer-specific survival.

Conclusions Ductal adenocarcinoma was more aggressive and had higher VASH1 expression than acinar adenocarcinoma, although MVD was equivalent. These results indicate that VASH1 expression may serve as

\section{Strengths and limitations of this study}

- The discovery of novel and reliable biomarkers for diagnostic purpose or prognostic prediction for ductal adenocarcinoma of the prostate is the focus of much research because specific immunohistochemical biomarkers for ductal adenocarcinoma have not been established due to its rarity.

- To our knowledge, this study is the first to evaluate the level of vasohibin-1 (VASH1) expression, which is an endogenous angiogenesis regulator, in patients with ductal adenocarcinoma and compare the oncological outcome between ductal and acinar adenocarcinoma.

- Comprehensive linked clinicopathological and outcome data were available for all patients.

- Because of the limited sample size (due to the rarity of ductal adenocarcinoma) and the heterogeneous patient population, the findings of the study cannot be generalised.

a novel biomarker for the aggressive nature of ductal adenocarcinoma.

\section{INTRODUCTION}

Ductal adenocarcinoma of the prostate is a rare morphological subtype, and its pure and mixed with acinar adenocarcinoma types occur in less than $1 \%$ and up to $5 \%$ of patients with prostate cancer, respectively, while the majority have the acinar adenocarcinoma type. ${ }^{1}$ Histologically, ductal adenocarcinoma is characterised by the presence of tall, pseudostratified columnar epithelium with abundant cytoplasm with a papillary or cribriform architecture. ${ }^{2} 3$ In general, pure ductal adenocarcinoma is assigned with a Gleason score of $4+4=8$, as its prognosis has been shown to be similar to that of acinar adenocarcinoma of the same grade. ${ }^{134}$ 
However, ductal adenocarcinoma is often diagnosed at an advanced clinical stage with local or distant metastasis, making prostate-specific mortality significantly worse than that of acinar adenocarcinoma. ${ }^{5}$ Due to its rarity, ductal adenocarcinoma is poorly characterised immunohistochemically and genetically, and specific immunohistochemical biomarkers have not been established. ${ }^{16}$ The discovery of novel biomarkers for diagnostic significance or prognostic prediction and the identification of new targets for pharmacotherapy for ductal adenocarcinoma are, therefore, the focus of much research.

Angiogenesis is a critical biological process, which consists of neovascularisation. Angiogenesis is induced under certain pathological conditions such as wound healing, inflammation and cancer. Under normal conditions, angiogenesis is maintained by a complex balance of endogenous angiogenesis-stimulating and angiogenesisinhibiting factors. The microenvironment of prostate cancer includes reactive stroma, comprised of multiple cell types which have been altered from their normal state to become permissive of cancer cell progression. ${ }^{78}$ During tumourigenesis, the angiogenic switch entails new blood vessels sprouting either directly by induction of growth factors, such as vascular endothelial growth factor (VEGF) and transforming growth factor-beta, or indirectly by recruitment of host immune cells. Newly formed microvessels provide oxygen and nutrients, promoting tumour growth, malignant progression and metastasis formation. ${ }^{910}$

One of the biomarkers that could reflect intratumoral angiogenic aggressiveness is the microvessel density (MVD) $;{ }^{112}$ however, MVD may not reflect the angiogenic activity alone because it corresponds to the number of formed vessels, including quiescent ones. ${ }^{13}$ Vasohibin-1 (VASH1) is a novel endogenous angiogenesis regulator, which is specifically expressed in activated vascular endothelial cells (ECs). VEGF induces VASH1 via the VEGF receptor 2 and its downstream protein kinase C-d. ${ }^{14}{ }^{15}$ When ECs are exposed to VEGF, ECs increase their expression of VASH1 mRNA and VASH1 protein synthesis over a 24-hour period. VASH1 is induced at the transcriptional level for angiogenesis inhibition or at the post-transcriptional level for stress tolerance of ECs, and these functions are critical for the maintenance of vascular homeostasis. ${ }^{16}$ Previous studies have demonstrated that high VASH1 expression is a poor prognostic factor in several cancers, including those of the urinary organs. ${ }^{17-21}$ We have previously reported that high VASH1 expression is a poor prognostic indicator of disease progression in patients with localised acinar adenocarcinoma of the prostate. ${ }^{13}$

In the present study, we examined the expression of VASH1 and MVD using transurethral resection of the prostate (TURP) or radical prostatectomy (RP) samples in patients with rare ductal adenocarcinoma and acinar adenocarcinoma with a Gleason score of $4+4$. This study aimed to compare the level of VASH1 expression between ductal and acinar adenocarcinoma and investigate whether ductal adenocarcinoma possesses an aggressive angiogenesis behaviour and if it is related to clinical outcomes.

\section{METHODS \\ Patients}

We retrospectively analysed the clinical records of 1495 patients who underwent RP or TURP at two of our hospitals for the past 15 years. All pathological specimens were re-reviewed by dedicated uropathologists to unify the reproducibility of the diagnosis. At first, we excluded the intraductal carcinoma component for ductal adenocarcinoma. We exclusively extracted acinar adenocarcinomas with a Gleason score of 4+4; even adenocarcinomas with a minor amount of Gleason pattern 3 or 5 were excluded. In addition, we excluded the three following groups of patients: First, patients that had received neoadjuvant hormonal treatment before RP or TURP. Second, patients that were followed up at a different hospital. Third, patients who missed the follow-up appointment within the 6 -month window after the primary treatment. The remaining 14 patients diagnosed with ductal adenocarcinoma and 20 patients diagnosed with acinar adenocarcinoma with a Gleason score of $4+4$ were included. The clinical characteristics of patients are shown in table 1.

\section{Immunohistochemistry}

Tissue microarray blocks were constructed from formalinfixed paraffin-embedded RP and TURP specimens from all 34 patients. For each specimen, two cores with a diameter of $4 \mathrm{~mm}$ were obtained from the two target areas of the tumour that showed the most aggressive or predominant pattern of the acinar adenocarcinoma or from the ductal area, if present. Sections with $4 \mu \mathrm{m}$ thickness were cut from the tissue microarray blocks.

We performed immunohistochemical staining for VASH1 and CD34 as previously reported. ${ }^{13} 22$ First, tissue sections were deparaffinised in xylene, hydrated by immersion in graded alcohols, and then distilled water. After antigen retrieval, endogenous peroxidase activity was blocked using $0.3 \%$ hydrogen peroxidase. The tissue sections were then incubated with a blocking solution containing $6 \%$ dry milk in phosphate-buffered saline (PBS). The primary antibodies used were mouse monoclonal antibodies (mAbs): anti-human VASH1 mAb diluted at a concentration of $4 \mu \mathrm{g} / \mathrm{mL}$ and anti-CD34 (Nichirei Biosciences, Tokyo, Japan). Mouse mAb was used against a synthetic peptide corresponding to the 286-299 amino acid sequence of VASH1, as suggested by Watanabe $e t a l .{ }^{15} \mathrm{Next}$, the tissue sections were incubated with secondary antibodies (Histofine Simple Stain MAX PO (M); Nichirei Biosciences) after washing with PBS. The colour was developed with 3, 3-diaminobenzidine tetrahydrochloride in $50 \mathrm{mM}$ Tris- $\mathrm{HCl}(\mathrm{pH} 7.5)$ containing $0.005 \%$ hydrogen peroxide. Finally, the sections were counterstained with haematoxylin. The positive control slide CD34 antigen was prepared from paraffin-embedded 
Table 1 Clinicopathological characteristics in 34 patients with ductal adenocarcinoma and acinar adenocarcinoma

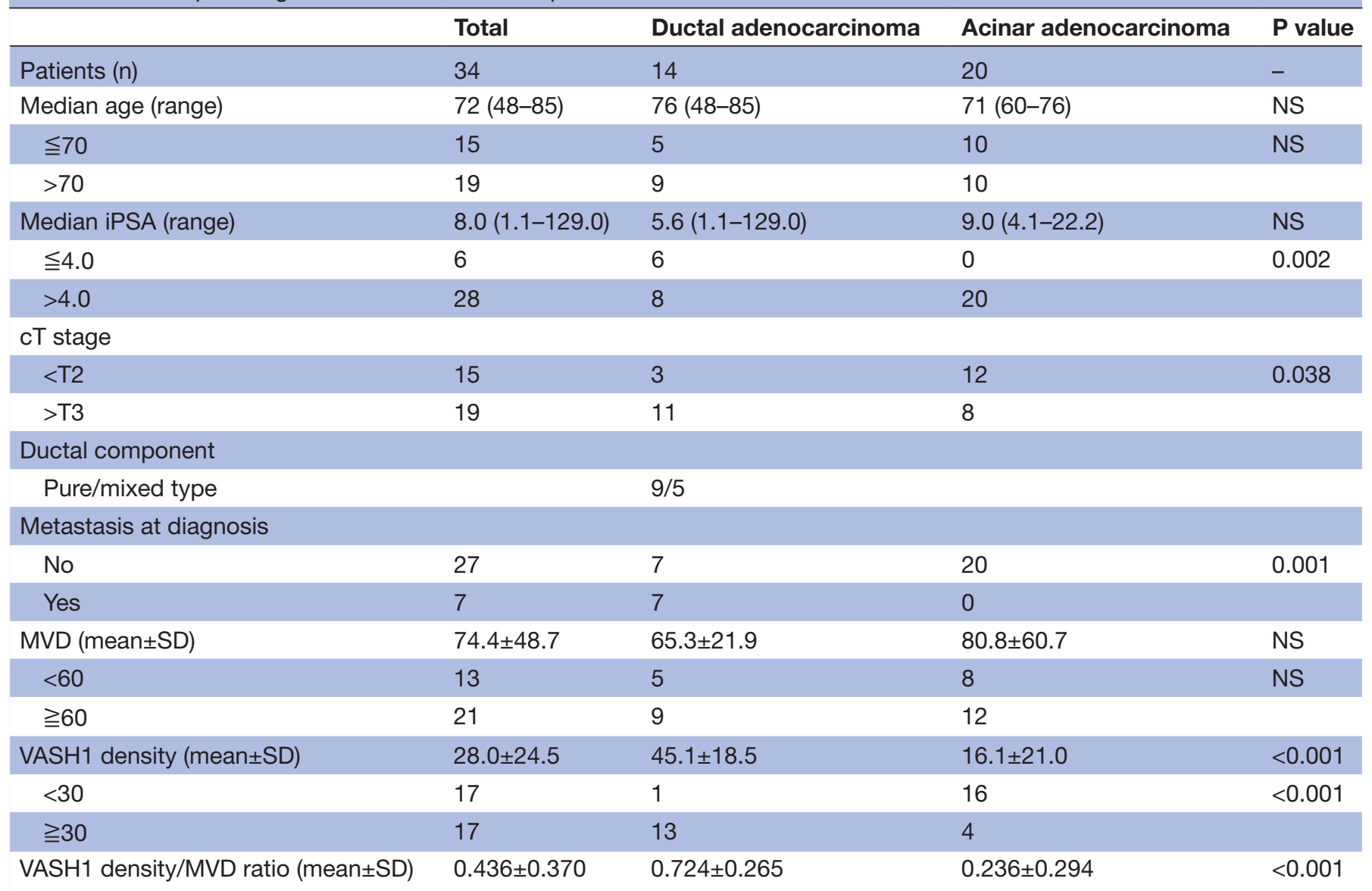

CT stage, clinical T stage; iPSA, initial prostate-specific antigen; MVD, microvessel density; NS, not significant; VASH1, vasohibin-1.

bladder cancer tissues with high MVD. Appropriate negative control slides for CD34 antigen and VASH1 were prepared by substituting the primary antibody with the immune globulin fraction of non-immune mouse serum at the same concentration in each staining run.

\section{Evaluation of immunostaining}

Two authors (HK and TKo) independently evaluated the immunoreactivity by counting the microvessels in proximity to the tumour using an Olympus IX71 microscope (Olympus, Tokyo, Japan). These authors were blinded to the clinical course of the patients, and the average of the numbers counted by the two investigators was used for subsequent analyses. The microvessels were identified based on their architecture, lumen lined by ECs and complemented by positivity of the ECs for anti-CD34 after scanning the immunostained section at low magnification $(\times 40$ and $\times 100)$. The areas with the highest number of distinctly highlighted microvessels were selected and counted at high magnification $(\times 200)$. Any immunostained EC or cluster separated from the adjacent vessels was counted as a single microvessel, even in the absence of the vessel lumen. Each count was defined as the highest number of microvessels identified at the hotspot. The highest number of microvessels in the hotspot was counted to determine MVD. The VASH1-positive signals were counted in the hotspot area, in which the highest number of vessels positive for anti-CD34 was identified. We defined the number of VASH1-positive signals per $\mathrm{mm}^{2}$ as the VASH1 density $\left(\right.$ vessels per $\left.\mathrm{mm}^{2}\right){ }^{21}$

\section{Statistical analysis}

The $\chi^{2}$ or Mann-Whitney U test was used to compare the VASH1 density or MVD of each location between patients with ductal and acinar adenocarcinoma. We used a median VASH1 density of $\geq 30 / \mathrm{mm}^{2}$ and a MVD of $\geq 60 / \mathrm{mm}^{2}$ as the cut-off level. The probability of cancerspecific survival (CSS) was estimated using the KaplanMeier method, and the median follow-up was calculated for patients who were alive at their last follow-up session. Differences among groups were considered significant at $\mathrm{p}<0.05$. These analyses were conducted using SPSS V.26.0 (IBM).

\section{Patient and public involvement}

Patients were not directly involved in the design or evaluation of the current report. 


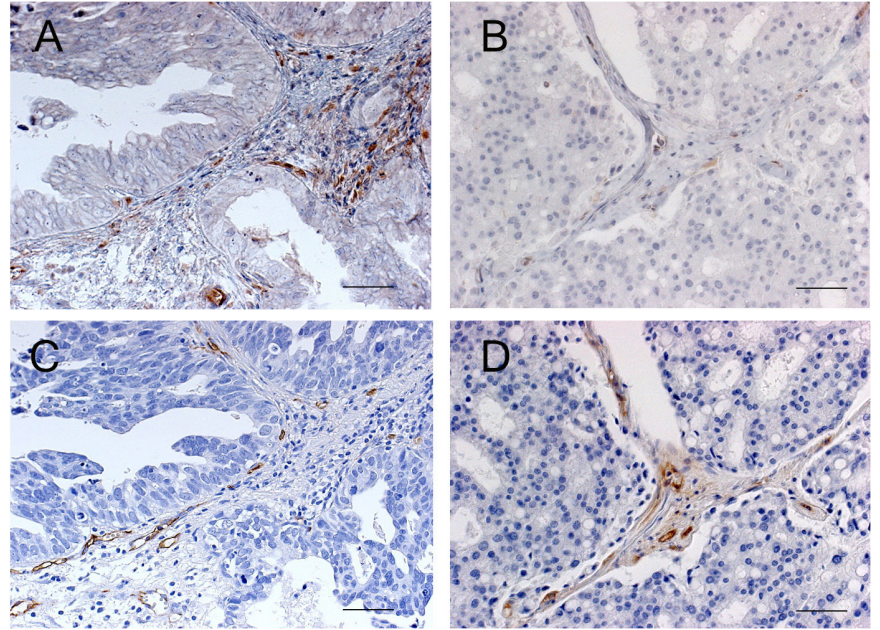

Figure 1 Immunostaining for VASH1 (vasohibin-1) and CD34 in ductal adenocarcinoma ( $A$ and $C$, respectively) and in acinar adenocarcinoma with the Gleason score of $4+4$ (B and $D$, respectively). Scale bar $=0.1 \mathrm{~mm}$.

\section{RESULTS}

\section{Patient characteristics and evaluation of VASH1 density and} MVD

The clinicopathological parameters and VASH1 density or MVD of 14 ductal adenocarcinoma and 20 acinar adenocarcinoma cases are shown in table 1 . The median patient age was 72 years (range 48-85 years) and the median pretreatment prostate-specific antigen (PSA) level was $8.0 \mathrm{ng} / \mathrm{mL}$. The pretreatment PSA level in patients with ductal adenocarcinoma (median $5.6 \mathrm{ng}$ / $\mathrm{mL}$, range $1.1-129.0 \mathrm{ng} / \mathrm{mL}$ ) was lower than that of patients with acinar adenocarcinoma (median $9.0 \mathrm{ng}$ / $\mathrm{mL}$, range $4.1-22.2 \mathrm{ng} / \mathrm{mL}$ ), and the levels varied widely. According to the pathology reports, nine patients (64.3\%) with ductal adenocarcinoma had a pure type, while five patients $(35.7 \%)$ had mixed ductal and acinar adenocarcinoma type. No statistically significant differences in clinical characteristics were noted between patients with pure and mixed types. Table 1 shows that the tumour of patients with ductal adenocarcinoma had a more aggressive behaviour than that of those with acinar adenocarcinoma. Nine patients $(64.3 \%)$ with ductal adenocarcinoma were diagnosed at an advanced clinical stage with local and distant metastases, and five patients $(35.7 \%)$ died of cancer during a median follow-up of 56.0 months.

To elucidate the biological significance of VASH1 in ductal adenocarcinoma, we examined the immunohistochemical expression of VASH1 and CD34 in the tumour tissue (figure 1). High VASH1 expression was observed in vascular ECs around the papillary architecture of tumours in ductal adenocarcinoma (figure 1A), while it was only partially observed in acinar adenocarcinoma lesions with a Gleason score of $4+4$ (figure $1 B$ ). The mean \pm SD VASH1 densities of ductal and acinar adenocarcinoma samples were $45.1 \pm 18.5$ vs $16.1 \pm 21.0 \quad(\mathrm{p}<0.001)$, respectively (table 1). On the other hand, the expression of CD34 in vascular ECs was at the same level (figure 1C,D), and the mean MVD of ductal and acinar adenocarcinoma samples were $65.3 \pm 21.9$ vs $80.8 \pm 60.7$ ( $\mathrm{p}=0.666)$, respectively.

We previously evaluated VASH1 expression levels in patients with acinar adenocarcinoma in two studies: one with 167 patients using RP specimens and the other with 104 patients using prostate needle biopsy specimens. The mean VASH1 densities were $9.9 \pm 7.3$ and $9.7 \pm 9.5$, respectively. ${ }^{13} 22$ Compared with previous data, the VASH1 density of acinar adenocarcinoma in the present study cohort was relatively higher because of the high Gleason score. Nevertheless, the VASH1 density of ductal adenocarcinoma samples was higher than that of acinar adenocarcinoma samples in this study.

\section{Prognostic significance of VASH1 expression}

Univariate analyses were performed to determine indicators of CSS. Figure 2 shows that ductal adenocarcinoma and high VASH1 expression were significant predictors of CSS (figure 2A,B), while high MVD was not a significant predictor (figure 2C). The 5-year CSS rates for high and low VASH1 expression were $70.0 \%$ and $100.0 \%$, respectively ( $\mathrm{p}=0.006)$. In addition, a low PSA level (PSA $<4.0 \mathrm{ng}$ / $\mathrm{mL}$ ) at the initial diagnosis (online supplemental figure 1) was a significant predictor of CSS. Multivariate analysis revealed no independent predictors of CSS.

Next, we focused on the differences in the VASH1 density/MVD ratio. The mean VASH1 density/MVD ratios for ductal and acinar adenocarcinoma samples were $0.724 \pm 0.265$ and $0.236 \pm 0.294(\mathrm{p}<0.001)$, respectively (table 1). Then, we re-evaluated the univariate analysis of
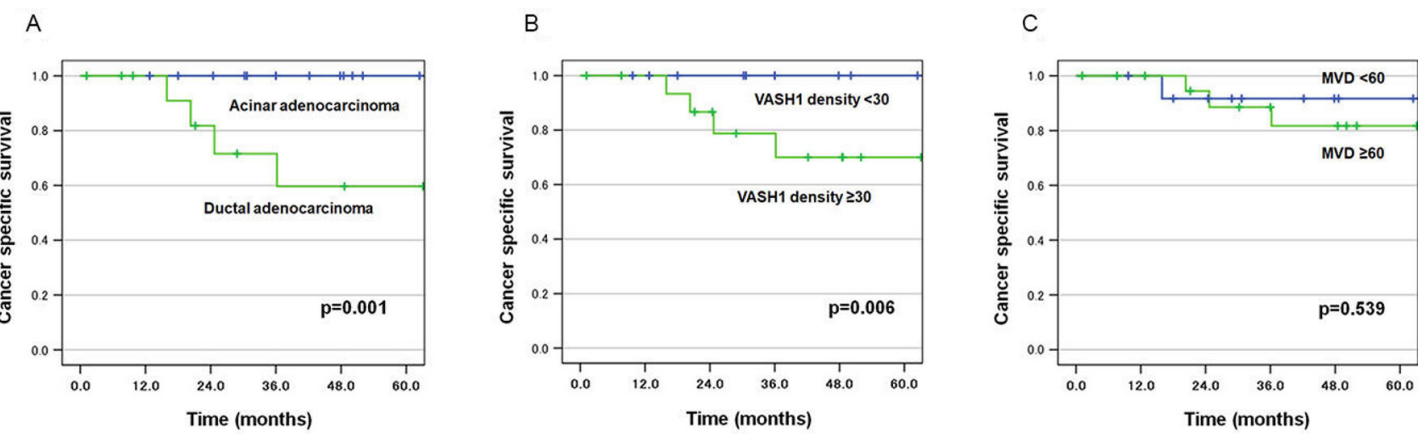

Figure 2 Kaplan-Meier curves of cancer-specific survival according to (A) histology, (B) microvessel density (MVD) and (C) vasohibin-1 (VASH1) density in all patients $(n=34)$. 
A

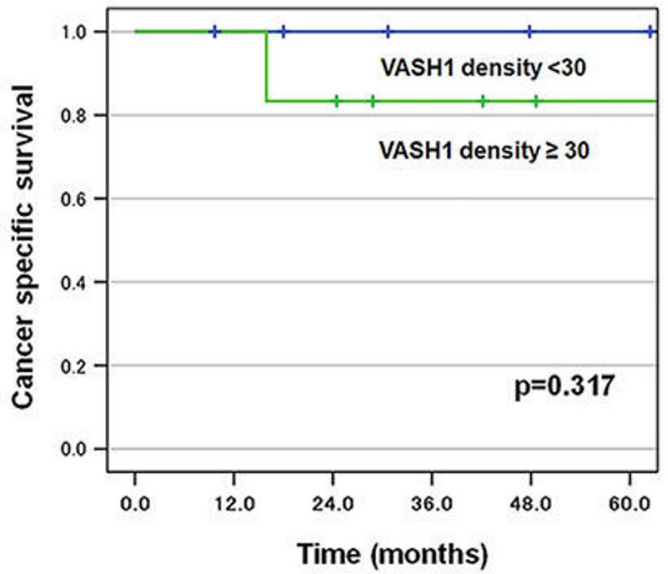

B

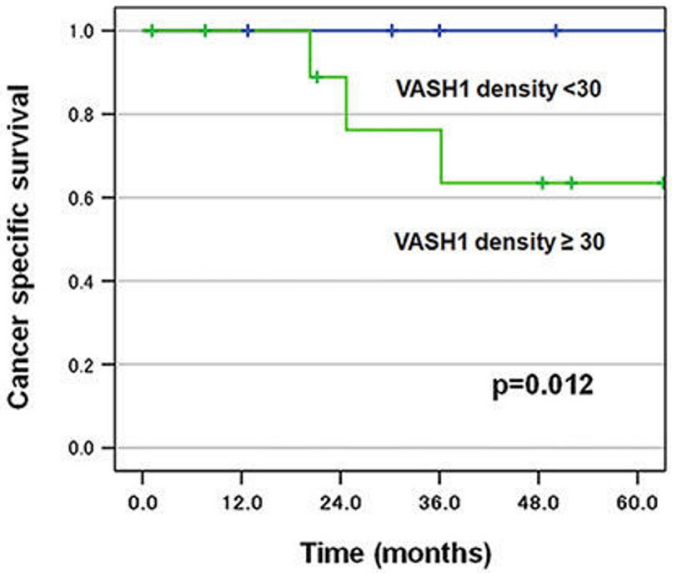

Figure 3 Kaplan-Meier curves of cancer-specific survival among (A) low microvessel density (MVD) groups ( $\mathrm{n}=13$ ) or (B) high MVD groups ( $\mathrm{n}=21$ ) according to the vasohibin-1 (VASH1) density.

VASH1 density separately for patients with low or high MVD values (figure 3). Six (46.2\%) and $11(55.0 \%)$ patients showed high VASH1 density in the low MVD group and high MVD group, respectively. The 5-year CSS rates for high and low VASH1 densities were $83.3 \%$ and $100.0 \%$ in the low MVD group $(\mathrm{p}=0.317)$ (figure $3 \mathrm{~A}$ ) and $63.5 \%$ and $100.0 \%$ in the high MVD group $(\mathrm{p}=0.012)$ (figure 3B). Together, these results demonstrated that MVD in ductal adenocarcinoma is not a predictor of CSS, but it could act as a complementary subdivision of the VASH1 density.

\section{DISCUSSION}

To the best of our knowledge, this study was the first to evaluate the level of neovascularisation around the tumour in patients with ductal adenocarcinoma using a novel angiogenesis regulator termed VASH1 and compared the oncological outcome between ductal and acinar adenocarcinoma. While the biological features along with the clinical and prognostic significance of ductal adenocarcinoma remain unclear, some studies have reported that ductal adenocarcinoma is usually detected at an advanced clinical stage, resulting in a higher rate of prostate cancerspecific mortality than acinar adenocarcinoma in general but similar to acinar adenocarcinoma with a Gleason score of 4+4. ${ }^{323}$ Ranasinghe et al reported that 112 $(25.7 \%)$ patients had metastasis at diagnosis among the largest cohort of 435 patients who were newly diagnosed with ductal adenocarcinoma and concluded that the rate of de novo metastatic ductal adenocarcinoma was much higher than that previously reported by other studies $(12.0 \%-13.6 \%) .^{25}$

In the era of PSA screening, early diagnosis and early radical treatment became increasingly possible, even in patients with high Gleason score or high-risk acinar adenocarcinoma in Japan. However, PSA screening tests are often ineffective in patients with ductal adenocarcinoma because they often present with only macroscopic haematuria and/or urinary obstruction without elevated serum PSA levels. This difficulty in early diagnosis may affect prognosis. In fact, 6 patients $(42.9 \%)$ with ductal adenocarcinoma showed a low PSA level $(<4.0 \mathrm{ng} / \mathrm{mL})$, 11 patients $(78.6 \%)$ had a cT3 stage or worse, and 7 patients $(50.0 \%)$ showed distant metastasis at the time of diagnosis in this study. Moreover, the oncological outcome of ductal adenocarcinoma was predominantly worse than that of acinar adenocarcinoma with a Gleason score of $4+4$

We hypothesised that increased angiogenesis is one of the main factors causing malignant progression and poor prognosis of ductal adenocarcinoma. In our previous study, we reported that since MVD corresponds to the number of formed vessels and included vessels without any potential for neovascularisation in acinar adenocarcinoma, VASH1 was expressed almost selectively in tumourassociated ECs. ${ }^{13} 15$ We also revealed that high VASH1 expression was associated with tumour malignancy as evaluated by the Gleason score and pT staging, and it was an independent predictor of tumour progression in acinar adenocarcinoma. ${ }^{1322}$ No previous studies quantitatively analysed the immunohistochemical evaluation of neovascularisation in ductal adenocarcinoma. Therefore, whether VASH1 expression and/or MVD offer prognostic information may add value to the diagnosis of ductal adenocarcinoma.

This contribution has, however, been studied in other lesions. For instance, in hepatocellular carcinoma (HCC), the prognostic role of MVD is controversial. Several studies have suggested that increased MVD could serve as an independent prognostic factor of decreased overall survival. $^{2627}$ On the other hand, several studies have demonstrated significantly lower MVD in patients with advanced-stage HCC. ${ }^{28}$ Accordingly, Murakami et al reported that VASH1-positive vessels had higher angiogenic activity than the CD34-positive ones in HCC. In addition, they found that the VASH1/CD34 ratio 
increased according to tumour progression and was associated with poor prognosis. ${ }^{30}$

In our VASH1 and CD34 staining, the ductal adenocarcinoma specimens showed high VASH1 expression irrespective of the serum PSA level, and this expression was predominantly higher than that of acinar adenocarcinoma specimens, while MVD showed no differences between the two lesions. In addition, the VASH1 density/ MVD ratio was higher in ductal adenocarcinoma than in acinar adenocarcinoma, and patients presenting with high MVD, and high VASH1 density likely have a worse prognosis. These results indicated that ductal adenocarcinoma showed more activated ECs with aggressive neovascularisation than acinar adenocarcinoma with a Gleason score of $4+4$, which had a pronounced growth and poor prognosis among all prostate cancers. Additionally, when we examined VASH1 expression in normal tissues adjacent to the cancer in several ductal adenocarcinoma patients, we found that VASH1 expression tended to be higher in ductal adenocarcinoma than in acinar adenocarcinoma. This result may reflect the cancer microenvironment of increase angiogenesis throughout the prostate in ductal adenocarcinoma. Since aggressive neovascularisation in ductal adenocarcinoma does not depend on the PSA level, the invasion or progression pattern of ductal adenocarcinoma may be different from that of acinar adenocarcinoma; in any case, the expression of VASH1 could serve as a novel biomarker for the aggressive nature of ductal adenocarcinoma.

In contrast, in patients with oesophageal squamous cell carcinoma, which is a fast-growing cancer with poor prognosis, Ninomiya et al reported that high VASH1 and VASH2 expression are predictors of poor prognosis. Subsequently, Yamamoto et al demonstrated that the plasma concentrations of VASH1 and VASH2 are associated with poor oncological outcomes in patients with oesophageal squamous cell carcinoma. ${ }^{19}$ (1) These new approaches and viewpoints can be considered for application to other cancers. In recent years, targeted drug therapy based on genomic sequencing or liquid biopsy has been widely used for several cancers. Liquid biopsy is the real-time analysis of tumour cells or tumour cell products released into the blood or other body fluids by primary or metastatic tumour lesions. ${ }^{32}$ These techniques can enable the development of new methods for the early detection of primary cancer, determination of therapeutic targets and discovery of progression mechanisms. Currently, although there are no established guidelines for ductal adenocarcinoma and its genomic or immunohistochemical profile remains unclear, large-scale immunohistochemical and/or genetic analyses as well as aggressive neovascularisation and its biomarker, VASH1, are expected to help determine ductal adenocarcinoma aggressiveness.

This study has several limitations. First, it was performed in a retrospective manner with a limited number of patients due to the rarity of ductal adenocarcinoma. The reason for the small number of patients with acinar adenocarcinoma with a Gleason score of $4+4$ is that the VASH1 density is much lower than that of ductal adenocarcinoma, as shown by our results, so we assumed that increasing the number of patients would not change the results. Second, the patient population was heterogeneous, with a short follow-up period. A larger multicentre study is, thus, warranted to confirm our findings. Finally, there were only a few treatment options or accurate imaging studies performed, such as multislice CT scan or multiparametric MRI, since the retrospective data collection involved a period over 10 years. This factor may adversely affect the analysis of the prognostic factors.

In conclusion, ductal adenocarcinoma specimens showed predominantly stronger VASH1 staining than those of acinar adenocarcinoma, and higher VASH1 expression was a significant predictor of CSS in patients with ductal adenocarcinoma. Overall, these results indicate that ductal adenocarcinoma possesses aggressive characteristics and that VASH1 could serve as a novel prognostic biomarker.

\section{Author affiliations}

${ }^{1}$ Department of Urology, Keio University School of Medicine, Tokyo, Japan ${ }^{2}$ Department of Urology, Saiseikai Yokohamashi Tobu Hospital, Yokohama, Japan ${ }^{3}$ Division of Diagnostic Pathology, Keio University School of Medicine, Tokyo, Japan ${ }^{4}$ Division of Diagnostic Pathology, Saiseikai Yokohamashi Tobu Hospital, Yokohama, Japan

${ }^{5}$ Department of Vascular Biology, Institute of Development, Aging and Cancer, Tohoku University, Sendai, Japan

Twitter Takeo Kosaka @takemduro

Acknowledgements We thank the members of the Division of Diagnostic Pathology, Saiseikai Yokohamashi Tobu Hospital; Ryutaro Mukouchi for technical assistance. We also thank the colleagues from the Department of Vascular Biology, Institute of Development, Ageing and Cancer, Tohoku University for their excellent technical assistance in purifying anti-human VASH1 mAb.

Contributors HK and TKo designed the study concept, analysed the data and wrote the manuscript. SM, TKi, HH and MK were responsible for data collection and interpretation of study results. YS supervised the research design and edited the manuscript. MO designed the study concept and supervised the manuscript. All authors read and approved the final manuscript. TKo is responsible for the overall content as the guarantor.

Funding The authors have not declared a specific grant for this research from any funding agency in the public, commercial or not-for-profit sectors.

Competing interests None declared.

Patient consent for publication Not applicable.

Ethics approval This study was approved by the ethics committees of the Keio University Hospital (\#20160084) and Saiseikai Yokohamashi Tobu Hospital (\#20200102).

Provenance and peer review Not commissioned; externally peer reviewed.

Data availability statement Data are available upon reasonable request.

Supplemental material This content has been supplied by the author(s). It has not been vetted by BMJ Publishing Group Limited (BMJ) and may not have been peer-reviewed. Any opinions or recommendations discussed are solely those of the author(s) and are not endorsed by BMJ. BMJ disclaims all liability and responsibility arising from any reliance placed on the content. Where the content includes any translated material, BMJ does not warrant the accuracy and reliability of the translations (including but not limited to local regulations, clinical guidelines, terminology, drug names and drug dosages), and is not responsible for any error and/or omissions arising from translation and adaptation or otherwise.

Open access This is an open access article distributed in accordance with the Creative Commons Attribution Non Commercial (CC BY-NC 4.0) license, which 
permits others to distribute, remix, adapt, build upon this work non-commercially, and license their derivative works on different terms, provided the original work is properly cited, appropriate credit is given, any changes made indicated, and the use is non-commercial. See: http://creativecommons.org/licenses/by-nc/4.0/.

\section{ORCID iD}

Takeo Kosaka http://orcid.org/0000-0002-4371-4594

\section{REFERENCES}

1 Seipel AH, Delahunt B, Samaratunga $H$, et al. Ductal adenocarcinoma of the prostate: histogenesis, biology and clinicopathological features. Pathology 2016;48:398-405.

2 Morgan TM, Welty CJ, Vakar-Lopez F, et al. Ductal adenocarcinoma of the prostate: increased mortality risk and decreased serum prostate specific antigen. J Urol 2010;184:2303-7.

3 Meeks JJ, Zhao LC, Cashy J, et al. Incidence and outcomes of ductal carcinoma of the prostate in the USA: analysis of data from the surveillance, epidemiology, and end results program. BJU Int 2012;109:831-4.

4 Epstein Jl. An update of the Gleason grading system. J Urol 2010;183:433-40.

5 Humphrey PA. Histological variants of prostatic carcinoma and their significance. Histopathology 2012;60:59-74.

6 Seipel $\mathrm{AH}$, Samaratunga $\mathrm{H}$, Delahunt $\mathrm{B}$, et al. Immunohistochemistry of ductal adenocarcinoma of the prostate and adenocarcinomas of non-prostatic origin: a comparative study. APMIS 2016;124:263-70.

7 McAlhany SJ, Ressler SJ, Larsen M, et al. Promotion of angiogenesis by ps20 in the differential reactive stroma prostate cancer xenograft model. Cancer Res 2003;63:5859-65.

8 Condon MS. The role of the stromal microenvironment in prostate cancer. Semin Cancer Biol 2005;15:132-7.

$9 \mathrm{Li} \mathrm{Y,} \mathrm{Cozzi} \mathrm{PJ.} \mathrm{Angiogenesis} \mathrm{as} \mathrm{a} \mathrm{strategic} \mathrm{target} \mathrm{for} \mathrm{prostate} \mathrm{cancer}$ therapy. Med Res Rev 2010;30:23-66.

10 van Moorselaar RJA, Voest EE. Angiogenesis in prostate cancer: its role in disease progression and possible therapeutic approaches. Mol Cell Endocrinol 2002;197:239-50.

11 Bono AV, Celato N, Cova V, et al. Microvessel density in prostate carcinoma. Prostate Cancer Prostatic Dis 2002;5:123-7.

12 de la Taille A, Katz AE, Bagiella E, et al. Microvessel density as a predictor of PSA recurrence after radical prostatectomy. A comparison of CD34 and CD31. Am J Clin Pathol 2000;113:555-62.

13 Kosaka T, Miyazaki Y, Miyajima A, et al. The prognostic significance of vasohibin-1 expression in patients with prostate cancer. $\mathrm{Br} J$ Cancer 2013;108:2123-9.

14 Shimizu K, Watanabe K, Yamashita H, et al. Gene regulation of a novel angiogenesis inhibitor, vasohibin, in endothelial cells. Biochem Biophys Res Commun 2005;327:700-6.

15 Watanabe K, Hasegawa $Y$, Yamashita $\mathrm{H}$, et al. Vasohibin as an endothelium-derived negative feedback regulator of angiogenesis. $J$ Clin Invest 2004;114:898-907.
16 Sato Y. Double-Face of vasohibin-1 for the maintenance of vascular homeostasis and healthy longevity. J Atheroscler Thromb 2018;25:461-6.

17 Miyazaki Y, Kosaka T, Mikami S, et al. The prognostic significance of vasohibin-1 expression in patients with upper urinary tract urothelial carcinoma. Clin Cancer Res 2012;18:4145-53.

18 Sano R, Kanomata N, Suzuki S, et al. Vasohibin-1 is a poor prognostic factor of ovarian carcinoma. Tohoku J Exp Med 2017;243:107-14.

19 Ninomiya Y, Ozawa S, Oguma J, et al. Expression of vasohibin-1 and -2 predicts poor prognosis among patients with squamous cell carcinoma of the esophagus. Oncol Lett 2018;16:5265-74.

20 Mikami S, Oya M, Kosaka T, et al. Increased vasohibin-1 expression is associated with metastasis and poor prognosis of renal cell carcinoma patients. Laboratory Investigation 2017;97:854-62.

21 Tamaki K, Moriya T, Sato Y, et al. Vasohibin-1 in human breast carcinoma: a potential negative feedback regulator of angiogenesis. Cancer Sci 2009;100:88-94

22 Kobayashi H, Kosaka T, Mikami S, et al. Vasohibin-1 as a novel microenvironmental biomarker for patient risk reclassification in lowrisk prostate cancer. Oncotarget 2018;9:10203-10.

23 Tu S-M, Lopez A, Leibovici D, et al. Ductal adenocarcinoma of the prostate: clinical features and implications after local therapy. Cancer 2009;115:2872-80.

24 Ranasinghe W, Shapiro DD, Hwang $\mathrm{H}$, et al. Ductal prostate cancers demonstrate poor outcomes with conventional therapies. Eur Urol 2021;79:298-306.

25 Ranasinghe WKB, Brooks NA, Elsheshtawi MA, et al. Patterns of metastases of prostatic ductal adenocarcinoma. Cancer 2020;126:3667-73.

26 Tanigawa N, Lu C, Mitsui T, et al. Quantitation of sinusoid-like vessels in hepatocellular carcinoma: its clinical and prognostic significance. Hepatology 1997;26:1216-23.

27 Sun HC, Tang ZY, Li XM, et al. Microvessel density of hepatocellular carcinoma: its relationship with prognosis. J Cancer Res Clin Oncol 1999;125:419-26.

28 Poon RT-P, Ng IO-L, Lau C, et al. Tumor microvessel density as a predictor of recurrence after resection of hepatocellular carcinoma: a prospective study. J Clin Oncol 2002;20:1775-85.

29 Ho J-W, Poon R-T, Sun C-K, et al. Clinicopathological and prognostic implications of endoglin (CD105) expression in hepatocellular carcinoma and its adjacent non-tumorous liver. World $J$ Gastroenterol 2005;11:176-81.

30 Murakami K, Kasajima A, Kawagishi N, et al. The prognostic significance of vasohibin 1-associated angiogenesis in patients with hepatocellular carcinoma. Hum Pathol 2014;45:589-97.

31 Yamamoto M, Ozawa S, Ninomiya Y, et al. Plasma vasohibin-1 and vasohibin-2 are useful biomarkers in patients with esophageal squamous cell carcinoma. Esophagus 2020;17:289-97.

32 Pantel K, Alix-Panabières $\mathrm{C}$. Circulating tumour cells in cancer patients: challenges and perspectives. Trends $\mathrm{Mol}$ Med 2010;16:398-406. 